Mit Hilfe dieses Pile-Simulators soll die für die Regulierung wichtige Übertragungsfunktion ermittelt werden. Sodann kann ein Modell für den Servomechanismus der Regulierung damit untersucht werden. Schliesslich ermöglicht die Untersuchung des zeitlichen Verhaltens der Neutronendichte bei verschiedenen Reaktivitätsänderungen die Aufstellung eines Programms für das Anlassen und Abstellen des Reaktors, was jeweils mit genügender Vorsicht zu geschehen hat.

\title{
Jahrestagung GAMM 1956
}

Die Gesellschaft für Angewandte Mathematik und Mechanik (GAMM) veranstaltet ihre nächste wissenschaftliche Jahrestagung in der Zeit vom 22. bis 26. Mai 1956 (Pfingstwoche) in Stuttgart. Die örtliche Tagungsleitung liegt in Händen von Professor Dr. Günther Schulz, Mathematisches Institut A der Technischen Hochschule Stuttgart. H. GÖRTLER

\section{Erratum}

Asymptotische Entwicklungen für Fresnelsche Integrale und verwandte Funktionen und ihre Anwendungsmöglichkeiten bei der Berechnung spezieller Raketenbahnen. VOn JOSEF ZBORNIK, Chur (ZAMP 5, Fasc. 4, $345[1954])$.

Wie P. Henrici (ZAMP 6, Fasc. 2, "Kleine Bemerkungen...") hervorhob, weist der Aufsatz Asymptotische Entwicklungen für Fresnelsche Integrale..., ZAMP 5, Fasc. 4, 345 ff. (1954), zwei Inkorrektheiten auf. Zu ihrer Berichtigung sind folgende Korrekturen vorzunehmen:

Es ist zu streichen: S. 347, 7. Zeile von unten: "... das Restglied..."

Es ist $z \mathrm{u}$ setzen:

S. 347,6 . Zeile von unten: $x^{n} R_{n}(x)$ anstatt $\left.R_{n}(x)^{1}\right)$.

5. Zeile von unten: $\lim _{x \rightarrow \infty} x^{n} \int_{x}^{\infty} \ldots$ ansta.tt $\lim _{x \rightarrow \infty} \int_{x}^{\infty} \ldots$

S. 348, 3. Zeile von oben: $\lim _{x \rightarrow \infty} x^{n} L_{1}$ anstatt $\lim _{x \rightarrow \infty} L_{1}$,

$$
\lim _{x \rightarrow \infty} x^{n} L_{2} \quad \text { anstatt } \quad \lim _{x \rightarrow \infty} L_{2} .
$$

4. Zeile von oben: $x^{n} R_{n}(x)$ anstatt $R_{n}(x)$.

S. 349, 10. Zeile von unten: "Da in den betrachteten...» anstatt "Da in ...»

1) Vgl. zum Beispiel: LeNse, Reihenentwicklungen in der mathematischen Physik, 2. Aufl. (Walter de Gruyter \& Co., Berlin 1947), S. 6. 